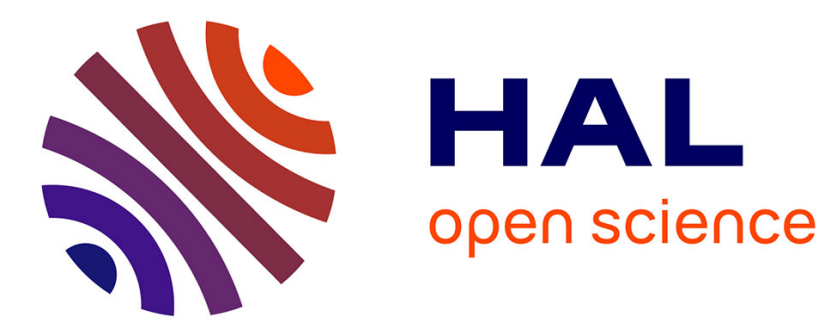

\title{
Lectures et mutations du paysage des marais bas-iraqiens aux premiers siècles de l'Islam \\ Noëmie Lucas
}

\section{To cite this version:}

Noëmie Lucas. Lectures et mutations du paysage des marais bas-iraqiens aux premiers siècles de l'Islam. Hypotheses, 2021, 22 (1), pp.209-219. 10.3917/hyp.181.0209 hal-03212298

\section{HAL Id: hal-03212298 \\ https://hal.science/hal-03212298}

Submitted on 30 Apr 2021

HAL is a multi-disciplinary open access archive for the deposit and dissemination of scientific research documents, whether they are published or not. The documents may come from teaching and research institutions in France or abroad, or from public or private research centers.
L'archive ouverte pluridisciplinaire HAL, est destinée au dépôt et à la diffusion de documents scientifiques de niveau recherche, publiés ou non, émanant des établissements d'enseignement et de recherche français ou étrangers, des laboratoires publics ou privés. 


\section{Lectures et mutations du paysage des marais bas-iraqiens aux premiers siècles de l'Islam}

NoËMie LuCAs*

La Batiha c'est tout un pays dans le pays d'Irak, quelque chose d'à part, installé entre ciel et terre, entre les vastes étendues d'eau libre, les îles et les roseaux en masses serrées, à peine trouées de quelques cheminements possibles pour les embarcations, les fondrières et les sols asséchés, sans parler des canaux et des digues, des villages aussi, regroupés sur des lambeaux de terre à peine exhaussés sur cette masse ambiguë, et qui ont pris le relais des vieilles installations humaines aujourd'hui noyées, dont on peut voir les vestiges, au fond du lac, quand l'eau est claire ${ }^{1}$.

Avec ces mots, dans son ouvrage intitulé La Géographie humaine du monde musulman, André Miquel proposait une description des marais du Bas-Iraq ${ }^{2}$. Cette région correspond au sud de la province, appelée $\operatorname{Iraq}^{3}$ à l'époque islamique, qui se distingue, dans ses limites géographiques, de l'État irakien actuel. Souvent désigné par le terme arabe Sawâd, qui renvoie à la

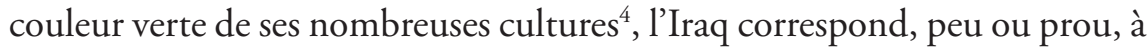
la Basse-Mésopotamie, et donc le Bas-Iraq au sud de celle-ci, soit à la plaine deltaïque qui s'étend entre le Tigre et l'Euphrate.

* Docteure en histoire, a soutenu, en décembre 2020, à l'université Paris 1 PanthéonSorbonne, une thèse intitulée "Le pouvoir de la terre : essai d'histoire de la société basiraqienne au $\mathrm{II}^{\mathrm{e}} / \mathrm{VIII}^{\mathrm{e}}$ siècle ", sous la direction d'Anne-Marie Eddé.

1. A. Miquel, La Géographie humaine du monde musulman jusqu'au milieu du XI' siècle, Paris, 1967-1988, t. 3, p. 203.

2. Voir la carte en annexe.

3. Afin de distinguer la province médiévale d'Iraq de l'État irakien actuel, je choisis deux orthographes différentes : Iraq pour la province, qui correspond à une translittération aménagée de l'arabe al-Irâq et Irak pour le pays contemporain.

4. La racine arabe s-w-d signifie d'abord " noir ", mais renvoie tout autant à la couleur verte, et donc aux nombreuses cultures présentes dans la région. Sur la parenté entre le noir et le vert, voir A. Morabia, "Recherches sur quelques noms de couleurs en arabe classique ", Studia Islamica, 21 (1964), p. 61-99 (ici p. 88-89). 
Le paysage bas-iraqien est globalement dominé par les eaux. Il est néanmoins pluriel, le nord se distingue du sud et du centre. Dans un même espace se côtoie une multiplicité de paysages, au nombre desquels il faut compter les marais, al-Batấih (sg. al-Batîha) dans les sources arabes, qu'André Miquel ramenait à la vie par son talent littéraire. Ce paragraphe, extrait de sa somme, est également le résultat d'un travail, celui de l'historien et de son enquête dans les sources, principalement celles produites par les géographes $\mathrm{du} \mathrm{X}^{\mathrm{e}}$ siècle.

Il s'agit, dans cet article, de tenter d'exposer les "processus de fabrication [d'une] recherche " qui se donne pour ambition de retrouver un paysage ancien. L'historien peut-il retrouver autre chose que les perceptions des témoins qui lui servent de sources ? Comment le paysage en tant que processus peut-il faire l'objet d'une recherche?

\section{Quand dire c'est ne pas décrire}

Dans une enquête sur les marais bas-iraqiens, deux types de sources peuvent être distingués : celles qui reviennent sur le processus de formation des marais et celles qui décrivent la région dans laquelle ils se situent, offrant en même temps aux lecteurs une description des marécages. Tandis que le premier ensemble de sources donne à lire les marais en mutation, donc dans le temps, le second propose plutôt un instantané de ceux-ci, à un «moment $\mathrm{T}$ », celui du $\mathrm{x}^{\mathrm{e}}$ siècle.

Parmi les auteurs arabes médiévaux qui reviennent sur la naissance des marécages, les principaux sont al-Balâdhurî (m. 892), al-Mas ûdî (m. v. 950) et Ibn Rusta, dont l'ouvrage a été composé entre la fin du Ix siècle et le début $\mathrm{X}^{\mathrm{e}}$ siècle $^{6}$. Dans les deux derniers cas, le récit de la formation des marais est inséré au sein de la description des fleuves de la région, en l'occurrence dans celle du Tigre, alors que dans le premier cas, Le Livre des conquêtes d'al-Balâdhurî, il se trouve dans une section spécifiquement consacrée aux

5. Cette expression est empruntée à É. AnHeim, «L'historien au pays des merveilles? Histoire et anthropologie au début du xxI siècle ", L'Homme, 203-204 (2012), p. 399-427 (p. 417). Cet article a inspiré la réflexion que nous proposons ici.

6. Al-Balâdhurî, Futûh al-Buldân, Beyrouth, 2014, p. 177-178; AL-Mas' ÛDî, Murûj ad-dhahâb, Beyrouth, 1996 (disponible en traduction française, Les Prairies d'or, Barbier de Meynard et Pavet de Courteille trad., Paris, 1989, t. 1, p. 224-226) ; Ibn Rusta, Kitâb al-a lâq an-nafîsa, M. J. DE Goeje éd., Leyde, 1892, p. 89-96. 
marais ${ }^{7}$, après le récit de la conquête de l'Iraq et de la Perse, et celui de la fondation des villes de Kûfa et de Wâsit.

Des objectifs littéraires différents justifient ces choix de compositions. Al-Mas ûdî et Ibn Rusta répondent à une visée encyclopédico-géographique. Ils proposent un savoir multiforme qu'ils rattachent au Tigre, dans la droite lignée de ce que l'on trouve dans les ouvrages de géographie mathématique, où la description des marais vient dans le prolongement de celle du fleuve. Al-Balâdhurî, quant à lui, opère un choix de composition original. Le récit se situe après des développements sur la ville de Wâsit. Il n'y a rien d'étonnant à cela puisque les marais/al-batẩ ì se situent en aval de cette ville. En revanche ce récit se singularise en ne respectant pas les découpages administratifs connus, et ce d'autant plus que les districts rattachés au Tigre, dont fait partie Basra, sont évoqués à un autre endroit de l'ouvrage.

La formation des marais trouve son origine à l'époque sassanide et est présentée comme un phénomène naturel et anthropique progressif. Parmi les principaux évènements rapportés, nous apprenons que l'eau rompit les digues insuffisamment entretenues par le roi sassanide Qubâdh ibn Fayrûz (r. 488-496 et 498-531). La dernière phase d'une longue évolution a lieu sous Chosroès II (r. 590-628), lorsque l'Euphrate et le Tigre connaissent une crue exceptionnelle et forcent leurs digues pour former alors le grand marécage appelé al-Batîha. Les derniers Sassanides tentent, en vain, de réparer les dommages ${ }^{8}$. Lors de la conquête de la région par les armées arabo-musulmanes, à partir de 636, "l'eau étendit ses ravages sans que l'on cherchât à y remédier, et les étangs gagnèrent chaque jour du terrain ${ }^{9}$ ». Ainsi prend fin le récit de la naissance de ce grand marécage, présenté comme le résultat de phénomènes naturels, liés aux aléas inhérents à ces grands fleuves aux cours changeants, ainsi qu'anthropiques, les digues manquant d'entretien du fait de la négligence humaine. Chez Ibn Rusta, le récit est différent, car il a pour vocation d'expliquer comment et en quoi le cours du Tigre fut modifié et quelles en furent les conséquences ${ }^{10}$ : ainsi, la brèche entraîna l'inondation de nombreux espaces cultivés mais aussi de villages,

7. Al-Balâdnurî, Futûh al-Buldân, op. cit., p. 177-178: la section est intitulée Amr al-Batâìh.

8. Ibid. ; AL-Mas' ûdî, Les Prairies d'or, op. cit., t. 1, p. 224-226 ; Ibn Rusta, Kitâb ala lâq an-nafisa, op. cit., p. 94-95.

9. AL-MAs' ÛDî, Les Prairies d'or, op. cit., p. 225.

10. Iвn Rusta, Kitâb al-a lâq an-nafisa, op. cit., p. 94-95. 
les parties aux plus basses altitudes furent intégralement recouvertes d'eau et les plus hautes devinrent des îles. Tandis qu'Ibn Rusta s'applique à présenter la description des conséquences physiques sur le paysage (disparition de villages, inondation d'espaces auparavant secs et/ou cultivés), al-Mas ûdî et al-Balâdhurî décrivent les étapes historiques de ce processus en insistant sur le rôle et la responsabilité de l'État sassanide.

Le second ensemble de textes - datant des $\mathrm{IX}^{\mathrm{e}}-\mathrm{X}^{\mathrm{e}}$ siècles - constitue une production littéraire de nature géographico-administrative. Il faut distinguer les ouvrages dits de géographie mathématique de ceux qui représentent une géographie dite "des itinéraires et des États ${ }^{11}$ ", tant dans leur structuration que dans leurs intentions. Un même ensemble de sources, proposant a priori des connaissances d'une même nature, ne donne pas la même lecture de ce paysage des marais bas-iraqiens. Ibn Hawqal dans Configuration de la terre ${ }^{12}$ et al-Muqaddasî dans La Meilleure Répartition pour la connaissance des provinces ${ }^{13}$ construisent leurs ouvrages en proposant une description région par région, au nombre desquelles se trouve l'Iraq. Dans ces deux ouvrages, les marais sont présentés comme faisant partie de la région. Cependant, alors que ces deux auteurs proposent des descriptions très détaillées du Khûzistân (région immédiatement à l'est de l'Iraq) et de l'Iraq, de manière générale, ils semblent renoncer, voire échouer, à décrire les marais. Ainsi, Ibn Hawqal évoque ces marais dans la légende/ explicitation de sa carte quand il cite les «marais de Basra ». Ils font partie du paysage de l'Iraq et, à ce titre, doivent être cités mais sans plus de détails. Al-Muqaddasî procède autrement. Il divise l'Iraq en six sections, chaque section comprenant une ville, sorte de capitale de district (Basra, Kûfa, Bagdad, Samarra, Wâsit, Hulwân) et il décrit les villes incluses dans chacune de ces sections. Les marais sont ainsi évoqués dans la section dont la principale ville est Wâsit, en lien avec les villes bordant ces marécages,

11. Renvoie à "al-masâlik wa-l-mamâlik». Pour des développements sur cette littérature géographique, voir A. Miquel, La Géographie humaine du monde musulman, op. cit., t. 1 et P. Verkinderen, Waterways of Iraq and Iran in the Early Islamic Period: Changing Rivers and Landscape of Mesopotamian Plain, Londres, 2013.

12. Ibn Hawqal, Configuration de la terre, J. H. Kramers et G. Wiet trad., Paris, 1964.

13. Al-Muqaddasî, Ahsan at-taqâsim fî márifat al-aqâlim, M. J. De Goeje éd., Leyde, 1887 ; AL-MuQaddasî, The Best Divisions for Knowledge of the Regions, B. Collins trad., Reading, 2001. 
al-Salîq et al-Jâmida ${ }^{14}$. Cette description est l'occasion pour lui d'un court développement sur les marais, tant d'un point de vue paysager (il évoque les étangs ou lacs, l'eau, les canaux, les terres cultivées) mais aussi climatique (la chaleur et l'humidité de l'air, caractéristiques des zones humides ${ }^{15}$ ). Il se plaît, par ailleurs, à quelques développements sur les habitants de cette région, signalant leurs habitudes alimentaires (consommation de poisson, chaleur de leur eau potable) et leur caractère (faiblesse d'esprit, langue corrompue) ${ }^{16}$. Il tient également des propos plus laudatifs en mettant en avant l'abondance de l'eau et des poissons et les qualités de guerriers des hommes de la région ainsi que leur maîtrise de la navigation dans ces eaux. Dans ces deux ouvrages, les marais sont donc présentés comme un des paysages de la région de l'Iraq qui occupent l'espace qui sépare Wâsit de Basra, mais ils n’y sont pas décrits précisément. On les nomme, on indique leur présence mais sans les délimiter, en offrant parfois aux lecteurs, comme dans le cas d'al-Muqaddasî, quelques mots sur les traits paysagers saillants - presque des topoï dans ce cas - et la vie des habitants de cet espace.

Dans son Livre des merveilles des sept climats $^{17}$, Suhrâb, auteur de la fin $\mathrm{du} \mathrm{Ix}^{\mathrm{e}}$ siècle et du début du $\mathrm{x}^{\mathrm{e}}$ siècle, propose un manuel pour la réalisation d'une mappemonde. Il reprend en partie l'ouvrage d'al-Khwârizmî (m. 850) Surat al-Ard, qui se divise en six sections : villes, montagnes, mers, îles, régions géographiques et rivières ${ }^{18}$. Aussi est-il question des marais à plusieurs reprises, à commencer par une mention dans le chapitre consacré aux lacs et marais. Ceux du Bas-Iraq sont nommés marais de Basra batîhat $a l-B a s r a^{19}$, ce qu'on lisait déjà dans la légende de la carte d'Ibn Hawqal. Ils sont présents dans la section consacrée à la description des canaux de Basra, des marais et du Tigre dit borgne, qui intervient après la présentation des

14. Al-MuQADDasî, Absan at-taqâsim fî márifat al-aqâlim, op. cit., p. 119 ; AL-MuQADDASî, The Best Divisions for Knowledge, op. cit., p. 108.

15. Ibid.

16. Ibid.

17. SurhâB, Kitâb 'ajẩib al-aqâlim as-sab'a, H. von Mzik éd., Leipzig, 1929. La partie concernant la Mésopotamie a été éditée et traduite par Guy Le Strange dans «Description of Mesopotamia and Baghdâd, Written about the Year 900 A. D. by Ibn Serapion. The Arabic Text Edited from a MS. in the British Museum Library, with Translation and Notes ", The Journal of the Royal Asiatic Society of Great Britain and Ireland, 27/1 (1895), p. 1-76 et 27/2 (1895), p. 255-315.

18. Al-KhwÂrizmî, Kitâb Sûrat al-ard, H. von Mzıк éd., Leipzig, 1926.

19. Surhầ, Kitâb 'ajẩib al-aqâlim as-sab'a, H. von Mzıк éd., p. 79. 
cours du Tigre et de l'Euphrate dans leur intégralité. Une grande partie de la description des marais consiste à en donner les délimitations ${ }^{20}$, ils apparaissent donc comme clairement identifiables par l'auteur. Pourtant, si l'on en croit Suhrâb, les marais ne se limitent pas à cet espace qu'il circonscrit. Ils sont en effet cités en de nombreux autres endroits dans son texte : que ce soit dans la description des canaux du Bas-Euphrate ou de ceux de Wâsit, on peut lire que ces derniers se déversent dans les marais ${ }^{21}$, de sorte que les marais sont présentés comme un "aboutissement " de toutes les eaux de l'Euphrate et des canaux de la rive occidentale du Tigre.

Cela correspond en effet assez bien à la représentation que l'on peut avoir de la Basse-Mésopotamie sur les cartes médiévales. Cependant, il apparaît que la sous-section consacrée à la description des marais ne décrit pas tous les marais mais seulement ceux qui sont désignés comme les marais de Basra. La zone des marais était en réalité bien plus étendue, mais l'auteur ne la décrit pas. Cet espace est d'autant moins décrit qu'il n'est pas répertorié dans la liste des marais qui se trouvait dans le chapitre consacré aux lacs et marais.

Que ce soit chez Ibn Hawqal, al-Muqaddasî ou Suhrâb, les marais sont présents, avec des développements plus ou moins importants. Pour autant, les auteurs échouent dans leur description. André Miquel le soulignait lorsqu'il écrivait :

[...] les auteurs ne perçoivent pas toujours avec la même lucidité le système dans son ensemble, faute d'une connaissance suffisante, ou d'une volonté qui ne reculerait pas devant les difficultés proposées, ou encore par l'effet d'un intérêt trop particulier porté à telle ou telle pièce du réseau ${ }^{22}$.

20. G. Le Strange, "Description of Mesopotamia », art. cité, arabe p. 28 et trad. p. 297. Les marais commençaient à al-Qatr et se terminaient au niveau du canal appelé Abû al-Asad. Cet espace de marais contenait trois lacs séparés par des détroits (ou plutôt chenaux-arabe zaqâq) de roseaux.

21. Par exemple G. Le Strange, "Description of Mesopotamia », art. cité, arabe p. 2021 et trad. p. 271 : «Il y a aussi, dérivé du Tigre, un canal que l'on nomme as-Sîb. [...]. Il traverse des villages et des domaines, passe par al-Jawâmid ; de nombreux autres canaux sont dérivés de lui. Il tourne et se jette dans les marais " (traduction en français de l'auteur).

22. A. Miquel, La Géographie humaine du monde musulman, op. cit., p. 200. 


\section{À la recherche des procédés de fabrication $d u$ discours sur les marais}

À bien des égards, les auteurs des sources convoquées pour cette recherche du paysage des marais bas-iraqien font figure de témoins d'un paysage disparu pour l'historien contemporain. Les relations de ce dernier avec le document historique sont ainsi placées sous le signe de la "séduction du témoignage ${ }^{23}$ ». S'il importe, non pas de s'en défaire, mais plutôt d'analyser cette attraction en partant à la recherche des démarches d'enquête de ces témoins, l'entreprise est malaisée lorsque les auteurs des sources revendiquent eux-mêmes leur posture de témoin « direct » et célèbrent l'enquête de terrain. Emmanuelle Tixier-Caceres a ainsi montré comment les géographes arabes, notamment al-Muqaddasî, font du témoignage direct la condition de possibilité de l'écriture de la géographie du monde musulman ${ }^{24}$. En fait de témoignage, Emmanuelle Tixier-Caceres précise quil faut l'envisager de manière plurielle car, pour ces géographes, « le témoignage des prédécesseurs est aussi valide, sinon davantage, que le témoignage acquis sur le terrain ${ }^{25} \%$.

Vraisemblablement, bien que les marais soient situés dans la province centrale de l'Empire abbasside, l'Iraq, les géographes ne semblent pas s'y être rendus. L'enjeu de l'accessibilité à ces marais est intimement lié à la nature de cet espace, malléable, mobile, dominé par l'eau. Les quelques développements sur ces marais confirment qu'ils représentaient des lieux à l'accès malaisé en raison des roseaux et des eaux peu profondes. En d'autres termes, les manières « de ne pas dire les marais » permettent en fait de saisir les spécificités de ce paysage. En ne circonscrivant pas l'espace de ces marais, les géographes s'appliquent donc à ne pas figer cet espace qui ne peut, de toute manière, pas l'être.

Pour qui écrivent ces auteurs ? Pour qui se font-ils d'abord les témoins ? Il est d'usage de dire de la géographie qu'elle était « la fille du califat » dans la mesure où elle était écrite pour les fonctionnaires, et où elle fournissait un savoir administratif. Or, la région des marais bas-iraqiens, depuis le milieu du $\mathrm{IX}^{\mathrm{e}}$ siècle au moins, échappait à l'autorité de l'État. Elle constituait une

23. É. Anheim, "L'historien au pays des merveilles ? ", art. cité, p. 406.

24. E. Tixier-CaCeres, " La valeur du témoignage dans la géographie arabe au Moyen Âge ", Hypothèses, 3 (2000), p. 82.

25. Ibid., p. 86. 
zone d'insécurité importante ${ }^{26}$, de brigandage et même de révolte ${ }^{27}$. En 869, par exemple, débuta la révolte des esclaves noirs appelés Zanjs. Cette révolte, qui se déroula dans le Bas-Iraq et notamment dans les marais dans lesquels les révoltés avaient trouvé refuge, marqua durablement la région. Aux siècles suivants, qui furent précisément ceux de la production de ces ouvrages de géographie, les marais devinrent même un État indépendan $\mathrm{t}^{28}$ et de petites dynasties locales comme les Banû Shâhîn contrôlèrent la région.

Les marais bas-iraqiens s'apparentaient ainsi, pour l'administration, à un espace de marges. En cela, ils répondent à ce que Gilles Deleuze et Félix Guattari conceptualisaient comme un espace lisse, par opposition à l'espace striée 29 . Parce qu'il est balisé, nommé, renommé aussi, compartimenté et territorialisé, que l'on peut y définir des itinéraires en se rendant d'un point $\mathrm{A}$ à un point $\mathrm{B}$, l'espace strié comprend tous les espaces bas-iraqiens saisis par le descripteur et codifiés par l'administration. Ce sont aussi les espaces sur lesquels s'exerce le pouvoir de l'État. À l'inverse, le propre de l'espace lisse est d'être insaisissable, ou bien saisissable seulement par point. Ce paysage est donc insaisissable aussi bien pour les géographes qui veulent le décrire et le délimiter que pour les administrateurs qui veulent le territorialiser.

C'est peut-être là que réside l'explication de la singularisation qu'opère al-Balâdhurî, lorsqu'il consacre à al-Batấih un chapitre en dehors des divisions administratives connues. Les marais constituaient un paysage à la marge de l'autorité de l'État qui, pour cette raison, faisait l'objet de projets d'investissements. Immédiatement après le récit de la formation des marais ou des grands marécages chez al-Balâdhurî, mais aussi al-Mas ûdî, suit un développement sur les différentes entreprises des califes omeyyades ${ }^{30}$ et leurs

26. Ibn Battûta (m. 770-779/1368-1377) écrit, par exemple, dans sa Rihla (récit de voyage) pour le XIv siècle : "Nous nous arrêtâmes dans une localité dite al-Idhar, dans une forêt de roseaux entourée d'eau ; elle est habitée par des bédouins connus sous le nom d'al-Ma'âdî : ce sont des brigands rafidites qui attaquèrent un groupe de derviches restés en arrière de la caravane [...]. " (Voyageurs arabes. Ibn Fadlân, Ibn Jubayr, Ibn Battûta et un auteur anonyme, P. Charles-Dominique éd., Paris, 1995, p. 529).

27. Voir notamment A. Popovic, La révolte des esclaves en Iraq au $\mathrm{III}^{e} / \mathrm{IX}^{e}$ siècle, Paris, 1976.

28. Voir l'article "al-Batîha » dans l'Encyclopédie de l'Islam, vol. 1, p. 1126-1130.

29. Conceptualisé dans G. Deleuze et F. Guattari, Mille plateaux. Capitalisme et Schizophrénie, Paris, 1980, notamment dans le chapitre "Traité de nomadologie : la machine de guerre".

30. Al-BalÂdhurî, Futûh al-Buldân, op. cit., p. 178 ; AL-Mas' Ûdî, Les Prairies d'Or, op. cit., t. 1, p. 225-226. 
subordonnés pour " récupérer» ou " gagner» des terres sur ces mêmes marais. $\mathrm{La}$ " récupération des terres » est rendue en arabe par le verbe istakhraja min qui signifie " extraire de ". Le processus de récupération est souvent décrit de la manière suivante : "En faisant couper les roseaux qui couvraient ces étangs et en refoulant l'eau à l'aide de digues et de barrières ${ }^{31}$. "

Les auteurs de ces sources se font donc, dans ce cas, les témoins des projets étatiques de revalorisation de ces espaces, menés certainement à des fins d'appropriation et de contrôle de ceux-ci. En participant à l'assèchement des marécages, l'État se fait, en même temps, producteur de paysage. Ainsi, le village nommé al-Jâmida ou al-Jawâmid, situé à proximité des marais ${ }^{32}$, est né de ces entreprises d'investissement, ce qui est confirmé tant par son toponyme ${ }^{33}$ que par ce qu'on lit de cette récupération chez al-Balâdhurî̀ ${ }^{34}$. La description des marais au $\mathrm{x}^{\mathrm{e}}$ siècle a été, dans une certaine mesure, façonnée et modifiée par l'État, qui a fait de ces marais, au cours des siècles précédents, l'espace privilégié de ses investissements. Ces entreprises sont d'autant plus soulignées dans les ouvrages qui les évoquent, que les auteurs écrivent à une période à laquelle ces marais ont commencé à échapper, voire avaient déjà échappé, au contrôle étatique.

Les manières de dire les marais, en ne les délimitant pas, en les décrivant à peine ou en rappelant simplement comment l'État tenta de récupérer des terres sur eux, sont tout aussi importantes que le contenu de ces divers descriptions ou récits pour l'historien qui cherche à saisir ce paysage. Ainsi, l'analyse de la production même des sources constitue un complément essentiel à l'utilisation de ces documents relatant d'abord la perception de ces témoins d'un certain paysage ${ }^{35}$.

31. Al-Balâdhurî, Futûh al-Buldân, op. cit., p. 178.

32. Al-Muqaddasî, Ahsan at-taqâsim fî ma'rifat al-aqâlim, op. cit., p. 119 et AL-MuQaddasî, The Best Divisions for Knowledge, op. cit., p. 108 ; Yầût, Mújâm al-Buldân, Beyrouth, 1977, vol. 2, p. 95-96.

33. La racine $\mathrm{j}-\mathrm{m}-\mathrm{d}$ en arabe signifie : épaissir, rendre dur. Le choix de nommer un village si proche des marais par ce toponyme ne peut pas être un hasard. Il indique le processus de récupération de terres décrit dans les sources historiques que recouvre le verbe arabe Istakhraja.

34. Al-BalÂdhurî, Futûh al-Buldân, op. cit., p. 178 : à propos du canal al-Janb, qui peut être vu sur les terres al-Jâmida "qui ont été récemment récupérées ».

35. Voir T. Bechini, G. Binois, N. Lucas et C. Stevanato, "Retrouver le paysage. Intérêts, sources et méthodes ", Hypothèses, 22 (2018), p. 165-172. 


\section{"Au moins demeure le paysage"?}

La démarche de l'enquête, que ce soit celle que nous entreprenons, celle d'André Miquel, et tout autant celle des géographes/descripteurs de ces marais, doit être prise en considération dans cette tentative de "retrouver un paysage ". L'introspection s'articule donc selon trois temporalités : la nôtre, qui tente de retrouver le paysage des marais bas-iraqiens des premiers siècles de l'Islam ; celle d'André Miquel, lorsqu'il composait sa Géographie humaine, et celle des descripteurs médiévaux, à la fois enquêteurs du $\mathrm{x}^{\mathrm{e}}$ siècle et témoins dans l'enquête.

Alors qu'il rappelait les limites de la description des géographes, André Miquel poursuivait en écrivant : "Au moins demeure le paysage ${ }^{36}$. " La citation servant d'exergue et d'accroche à cet article n'incarne pas autre chose. Conscient des enjeux propres aux modalités de lecture de ces textes, André Miquel offre la description d'une idée de ce paysage des marais tel qu'il était perçu par ses descripteurs. Il s'agit là du paysage en tant qu'il relève de l'appréhension visuelle. Il est peint par touches, pour reprendre l'idée d'un espace lisse. Parce qu'il est " quelque chose d'à part, installé entre ciel et terre ", ce n'est pas à sa dimension topographique que l'on s'attache mais à ses représentations paysagères. En cela, André Miquel est fidèle au discours des géographes et retrouve les représentations, sorte de constantes d'un paysage dont la nature est d'échapper à celui qui cherche à s'en saisir.

Une attention portée aux processus de fabrication de ces discours renseigne, quant à elle, sur le processus de production de ce paysage ainsi que sur les formes prises par sa description. Cela passe par l'analyse des modalités et des contextes de production des documents historiques, support de la démarche d'enquête historienne. Les éléments visuels qu'André Miquel a su retrouver, lorsque nous examinons les processus de leur fabrication et les objectifs des auteurs qui les utilisent, ne disent plus seulement les perceptions du paysage, mais font ressortir les processus de production de ce paysage, en particulier le rôle de l'État.

L'historien peut retrouver autre chose que les perceptions des témoins, auteurs des sources narratives qui dominent le corpus, lorsqu'il ne s'intéresse pas uniquement aux lectures de ces marais bas-iraqiens mais aussi à leur écriture. Les processus de fabrication de ces discours doivent être placés

36. A. Miquel, La Géographie humaine du monde musulman, op. cit., t. 3, p. 200. 
au cour de l'enquête car ils permettent d'expliquer les manières de dire et de ne pas circonscrire ces marais, mais aussi parce qu'ils rendent compte des mutations de ce paysage dont la mobilité et la malléabilité participent à sa définition.

\section{Annexe}

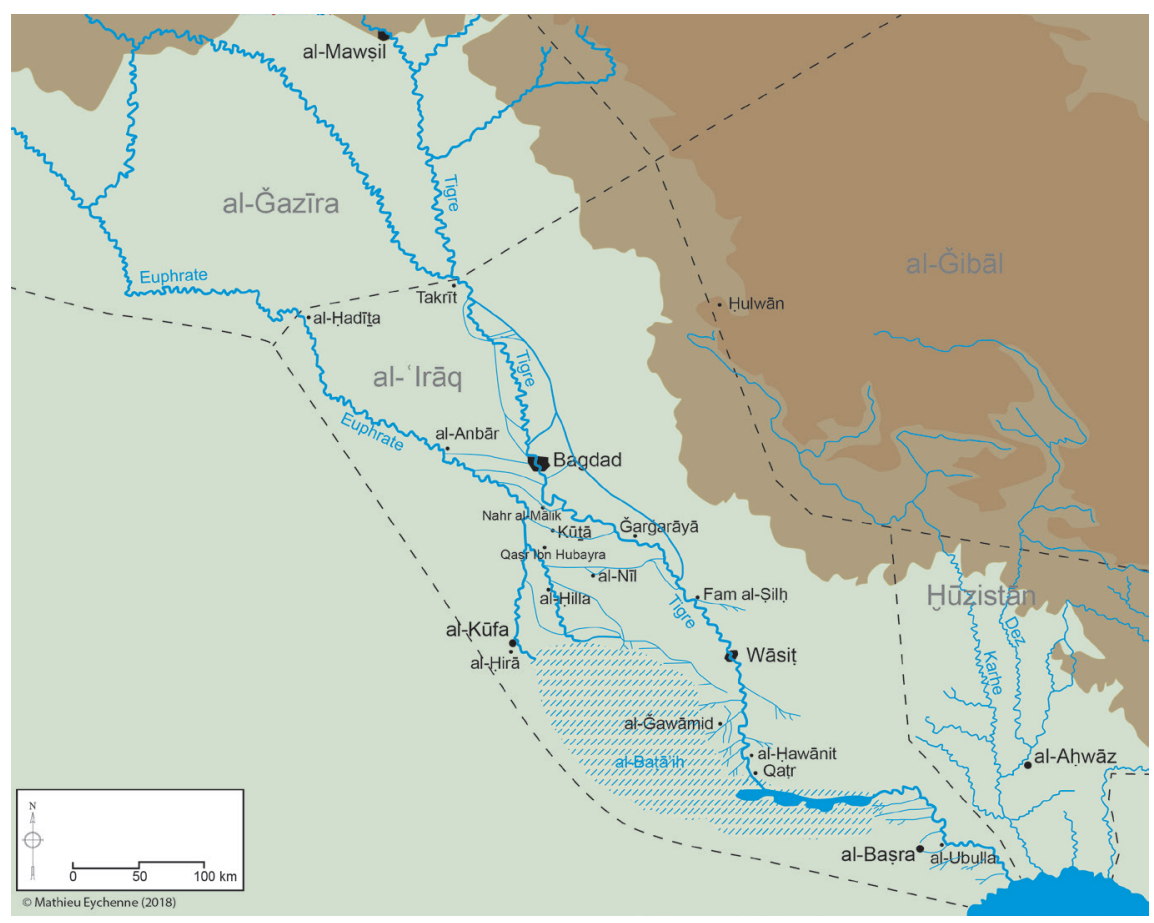

Le Bas-Iraq et ses marais à partir de la lecture des sources littéraires

Mathieu Eychenne, 2018. 
